\title{
The Mechanical Properties of Float Glass Surfaces Determined through Nanoindentation and Acoustic Microscopy
}

O. Goodman and B. Derby

School of Materials

University of Manchester

Oxford Road

Manchester, M13 9PL, UK

\begin{abstract}
We have combined nanoindentation with measurements of Rayleigh wave velocity, to determine the elastic constants of the surfaces of a commercial soda-lime float glass. The Young's modulus $(E)$ and Poisson's ratio $(v)$ of the glass surface exposed to air as: $E=80.5 \pm 0.5 \mathrm{GPa}$ and $v=0.20 \pm 0.01$, and for the surface in contact with the tin bath as: $E=77.3 \pm 1.0 \mathrm{GPa}$ and $v=0.19 \pm 0.015$. The difference in elastic constants is shown to be statistically significant. These measurements do not correlate with the expected changes in mechanical behaviour inferred from experiments on bulk glass specimens doped with tin, nor can they be explained by differences in defect density between the two surfaces.
\end{abstract}

\section{Keywords}

Acoustic methods; Nanoindentation; Non-metallic glasses (silicates); Elastic behaviour. 


\section{Introduction}

\subsection{Properties of Float Glass Surfaces}

Most flat soda-lime glass is manufactured using the float glass process. Molten glass is poured onto a liquid tin surface; on which it cools and solidifies, to produce two flat and parallel surfaces of a sheet with thickness typically in the range $2-10 \mathrm{~mm}$. These two surfaces will be subsequently distinguished as the tin side and air side of the glass sheet. After solidification, the continuous glass sheet undergoes a carefully controlled cooling process to eliminate any residual stresses before being cut into sheets for subsequent use. The air-side of the sheet remains free of any contact throughout this process. The tin-side has been in contact with liquid tin and there is some diffusion of tin into the glass structure near the interface. The tin side is also in contact with supporting rollers during heat treatment. Thus the two sides of the glass sheet undergo different histories during processing and it is the objective of this study to investigate whether these histories influence the mechanical properties of the glass surface.

It is well known that there is some incorporation of tin into the glass network structure during the float glass process. Depth profiling microanalysis using Rutherford backscattering (RBS) indicates that the highest concentrations of Sn occur in the top $100-200 \mathrm{~nm}$ of the tin side $[1,2]$. Sn can penetrate up to $40 \mu \mathrm{m}$ into the surface of float glass during processing where it exists in both stannous $\left(\mathrm{Sn}^{2+}\right)$ and stannic $\left(\mathrm{Sn}^{4+}\right)$ forms, with $\mathrm{Sn}^{2+}$ predominating near the surface and $\mathrm{Sn}^{4+}$ predominating in the subsurface region. Krohn et al [3] measured the elastic properties of bulk soda-lime glasses that had been doped with up to $3 \mathrm{~mol} \%$ tin oxide. They found a small increase in elastic modulus with increasing tin content. Ziemath et al found an increase in hardness 
of tin doped float glass with increasing Sn content and also a small increase in bulk density [4].

The thermal history of glass is known to influence its mechanical behaviour. The fictive temperature of a glass, $T_{f}$, is defined as the effective glass transition temperature of a glass for its particular thermal history. Glasses of identical composition, but with different $T_{f}$, can have significantly different physical properties. Vacher et al studied the properties of crown glass as a function of $T_{f}$ using Brillouin scattering and found a longitudinal sound velocity $c_{l}=5400 \mathrm{~ms}^{-1}$ at $T_{f}=740^{\circ} \mathrm{C}$ and $c_{l}=5600 \mathrm{~ms}^{-1}$ at $T_{f}=590^{\circ}$ C [5]. In another study, the influence of fictive temperature on specimen density was measured and the elastic modulus computed at two fictive temperatures for silica glass, with $c_{l}=5960 \mathrm{~ms}^{-1}$ and $E=78 \mathrm{GPa}$ at $T_{f}=1200^{\circ} \mathrm{C}$ and, $c_{l}=5980 \mathrm{~ms}^{-1}$ and $E=79 \mathrm{GPa}$ at $T_{f}=1500^{\circ} \mathrm{C}$ [6]. Thus if the air and tin sides of the glass have different values of $T_{f}$, they could have a measurable difference in their mechanical properties.

In addition, the tin side of the glass sheet passes over a number of solid roller surfaces during the manufacture of float glass. The surface of a brittle material is readily damaged through contact, introducing a population of sub-critical cracks. It is well known that the presence of a population of cracks within a body results in a reduction in its elastic properties. Warren et al published results on the mechanical properties of glass surfaces using acoustic microscopy and Brillouin scattering [7]. They found that the tin side showing a reduced elastic stiffness compared with the air side and they hypothesised that this was because the tin surface showed an increased level of damage. This was supported by a further study using Hertzian indentation that measured a significantly greater defect density on the tin side when compared with the air side [8]. 
Howell et al studied the tin and air sides of glass sheets using nanoindentation [9]. They reported small differences in the mechanical properties between the air and tin sides but concluded that these differences were not significant. They found a significant increase in elastic modulus and hardness when the top $150 \mathrm{~nm}$ of the glass surface was probed and suggested that this was caused by chemical differences between the near surface region and the interior of the glass. A more recent study by Kolluru et al reviewed the available literature on the mechanical properties of the tin and air surfaces of float glass and concluded that there was little consistency among the published data [10]. They varied the local environment through using cleaning procedures in acidic, neutral or alkali solutions prior to measuring the near surface elastic properties using nanoindentation. They found that the tin side of the glass was more resistant to chemical attack than the air side and they hence proposed that the poor agreement seen between published data is caused by poorly defined and documented experimental conditions; with environmental effects probably accounting for the differences in glass surface properties reported in the literature.

Thus we can see that the mechanical properties of the tin and air surfaces of float glass may be different because of their environment and history of processing; however prior studies have not conclusively determined whether this is so. It is the objective of this study to accurately quantify the elastic properties of the tin-side and air-side of float glass samples using two independent measures of near surface mechanical properties, nanoindentation and $z$-contrast acoustic microscopy, which measures the Rayleigh wave velocity on a surface, and thus determine whether there is any significant difference between them. 


\subsection{Nanoindentation}

Nanoindentation can be used to measure the mechanical properties of the near surface regions of materials through recording and interpreting the force displacement relation that occurs when a well defined indenter is driven into a surface. Oliver and Pharr [11] demonstrated that it is possible to analyse the force displacement data to derive an expression for the contact stiffness and hence the plane strain elastic modulus of a homogeneous and isotropic material,

$$
E^{*}=\frac{E}{\left(1-v^{2}\right)}
$$

where $E$ and $v$ are the elastic modulus and Poisson's ratio of the bulk material. When nanoindentation is used to characterize the elastic properties of a material, it has become common practice to assume a value for Poisson's ratio in equation 2 and to quote a calculated Young's modulus that is based on this assumption.

\subsection{Z-Contrast Acoustic Microscopy}

In an acoustic microscope, a piezoelectric transducer transmits high frequency acoustic waves through a sapphire lens. The lens is acoustically coupled to the surface through a drop of water. At the lens/water interface, acoustic waves are focussed to a spot at the water/material interface. Here two wave paths are important. One travels to the interface and is reflected straight back to the lens because of the mismatch in acoustic impedance between the water and the material. The second wave is refracted at the Rayleigh angle, $\theta_{R}$, and excites leaky Rayleigh waves along the specimen surface. These surface waves radiate acoustic energy in the form of a second acoustic wave back to the lens through the coupling fluid. The resulting signal at the lens is the interference 
between the reflected and radiated waves. As the lens is moved vertically out of focus the signal recorded as a function of displacement, $V(z)$, shows a characteristic series of oscillations the period of which, $\Delta z$, can be used to calculate the Rayleigh wave velocity, $v_{R}$, with [12]:

$$
\Delta z=\frac{v_{o}}{2 f\left(1-\cos \theta_{R}\right)}, \quad \text { with } \quad \sin \theta_{R}=\frac{v_{o}}{v_{R}}
$$

where $v_{o}$ is the velocity of sound in the coupling fluid (water) and $f$ is the acoustic frequency.

The velocity of the Rayleigh wave is related to the bulk longitudinal and transverse sound velocities in an (isotropic) solid medium, $v_{l}$ and $v_{t}$ respectively, with [13]

$$
\left(\frac{v_{R}}{v_{t}}\right)^{6}-8\left(\frac{v_{R}}{v_{t}}\right)^{4}+8\left(\frac{v_{R}}{v_{t}}\right)^{2}\left\{3-2 \frac{v_{t}^{2}}{v_{l}^{2}}\right\}-16\left\{1-\frac{v_{t}^{2}}{v_{l}^{2}}\right\}=0
$$

Equation 3 has only one root that is real and positive, giving a single value of $v_{R} / v_{t}$ for any given value of $v_{t} / v_{l}$. In an isotropic medium, the longitudinal and transverse sound velocities can be defined in terms of the elastic properties of the medium with

$$
\begin{gathered}
v_{l}=\sqrt{\left(\frac{E(1-v)}{\rho(1+v)(1-2 v)}\right)} \\
v_{t}=\sqrt{\left(\frac{E}{\rho 2(1+v)}\right)}
\end{gathered}
$$

where $\rho$ is the density of the medium. Equations $4 \mathrm{a}$ and $\mathrm{b}$ can be rearranged to give expressions for Poisson's ratio and Young's modulus with 


$$
\begin{gathered}
E=\frac{\rho v_{t}^{2}\left(3 v_{l}^{2}-4 v_{t}^{2}\right)}{\left(v_{l}^{2}-v_{t}^{2}\right)} \\
\frac{v_{t}}{v_{l}}=\sqrt{\left\{\frac{(1-2 v)}{2(1-v)}\right\}}
\end{gathered}
$$

Thus it can be seen that if the Rayleigh wave velocity is determined experimentally, we can determine the elastic properties of the material as defined by a function of Young's modulus and Poisson's ratio.

A previous study [14] combined $z$-contrast acoustic microscopy and nanoindentation to characterize the elastic properties of thin films and provide two independent measurements of Young's modulus and Poisson's ratio (equation 1 and equation 5). This allows the exact values of these two parameters to be determined and, for an elastically isotropic material, provide a complete description of the elastic stiffness tensor.

\section{Experimental Method}

Float glass sheet of thickness $4 \mathrm{~mm}$, with nominal composition and mechanical properties as given in Table 1, was provided by Pilkington Technology Centre, Lathom, UK, with the tin and air sides identified. Glass surfaces were used in the as-received state after cleaning with an ethanol soaked swab to remove any contamination. In all cases the tin and air sides of the same glass specimen were identified and tested.

Nanoindentation was carried out using an XP nanoindenter with a Berkovich diamond indenter (Agilent Technologies, USA). The system has load and displacement resolutions of $50 \mathrm{nN}$ and $<0.02 \mathrm{~nm}$, respectively. All experimental procedures were 
carried out as per ISO 14577 [16] with a standard fused silica block used for calibration. For each specimen, 30 indentation experiments were carried out in a regular array across the surface. Elastic properties were measured as a function of depth using the continuous stiffness monitoring (CSM) technique. In this method the indentation is arrested and a small unload is carried out to determine the contact stiffness at a range of depths before reloading during the indentation experiment. It has been suggested that when CSM is used to measure the mechanical properties of metal specimens, there is an influence of strain rate that results in a difference in hardness values when compared with quasi-static nanoindentation [17]. However, it was explicitly stated that no effect is expected or was observed that influences the elastic properties measured using CSM. Indeed here we found that measurements made at depths of $500 \mathrm{~nm}$ using CSM and quasistatic measurement returned the same value of elastic modulus within experimental error.

Acoustic measurements were taken using a KSI 2000 scanning acoustic microscope (Krämer Scientific Instruments, Herborn, Germany) that was equipped with a $1 \mathrm{GHz}$ spherical lens and a $\mathrm{ZnO}$ transducer. Distilled water was used as a coupling fluid at room temperature. The acoustic signal as a function of distance from focus, $V(z)$, was measured either repeatedly in a single spot by finding focus and traversing away from focus, or was measured in 30 different locations defined as an array. In order to ensure compatibility between the nanoindentation and acoustic data, the wave speed was calibrated using the same standard silica block used to calibrate the nanoindenter. 


\section{Results}

\subsection{Nanoindentation}

Figure 1 shows the plane strain Young's modulus $\left(\underline{E^{*}}\right)$ and hardness, obtained by nanoindentation, as a function of depth for the air side and tin side of a $4 \mathrm{~mm}$ sheet of float glass. In each case, thirty separate indents were made using the nanoindenter in continuous stiffness measurement mode. The error bar on the mean datum value in the figures shows the standard deviation of the test results. Both the elastic modulus and hardness data show similar trends as a function of indentation depth. For both surfaces the data shows a marked size effect with a considerable increase in elastic modulus and hardness at indentation depths $<200 \mathrm{~nm}$, this increase is also accompanied by an increase in the experimental scatter. At greater indentation depths the mechanical properties of the two sides deviate. In all cases at depths greater than $600 \mathrm{~nm}$, the values for Young's modulus and hardness become more consistent and the standard deviation of the modal value reduces presenting as a flat "plateau" region on the graphs. It can be seen that at these indentation depths the value of Young's modulus is slightly lower (an approximate difference of $3 \mathrm{GPa}$ or $4 \%$ throughout) for the tin-side of the float glass than for the air-side. This difference is several multiples of the standard deviation of the data and is thus statistically significant.

\subsection{Acoustic Microscopy}

Table 2 shows the results of the Rayleigh wave velocity measurements from the air side and tin side of a $4 \mathrm{~mm}$ thick float glass specimen. The data is grouped into two sets, each of 30 measurements. One set is taken from repeated measurements of the same spot on the surface and the second is taken from 30 measurements made in a $5 \times 6$ array with approximately $1 \mathrm{~mm}$ between measuring sites. The mean Rayleigh wave 
velocity $v_{R}$, has been calculated together with the standard error of the mean for each of these data sets. In view of the difference between the data sets being close to the standard error, Student's $T$-test was used to determine the significance of the difference between the results obtained from the two sides of the glass. The data in Table 2 displays 118 degrees of freedom and returns a T-statistic of $T_{118}=2.56$ and $P=1.15 \mathrm{x}$ $10^{-2}$, this indicates a statistically significant difference between the Rayleigh wave velocity measurements for the two sides of the glass sheet.

A lower Rayleigh wave velocity indicates a lower value of the Young's modulus of the surface region (equation 5), assuming that the density of the material remains unchanged. Using the assumed value for Poisson's ratio of 0.23 and the density, $\rho=$ $2530 \mathrm{kgm}^{-3}$ [15], equations 3-5 can be solved to give Young's modulus values of $81.6 \pm$ 1.0 GPa and 78.8 $\pm 1.2 \mathrm{GPa}$ for the air- and-tin sides respectively. These values show the same trend as was found with the nanoindentation results, that Young's modulus of the air side is greater than that of the tin side.

The data in Table 2 shows greater variation between individual Rayleigh wave measurements made at the same location on a single specimen than is seen with the array data. One might reasonably expect measurements taken at a spot to have a narrower range than measurements taken at a variety of locations on a specimen's surface. It is not obvious why the opposite effect was observed, especially as all possible sources of operator introduced error are common to both sets of measurements. In all cases the lens was raised away from the surface and refocused between measurements. Possible sources of error or experimental scatter are: inaccuracies in finding the exact point of focus, error in determining the periodicity of the $V(z)$ signal, or the replenishment of the coupling fluid (which for the array was done by moving the 
stage into the "home" position between every measurement and applying with the acoustic microscope's own device). However, for the measurements at a single spot, the water was applied manually only when required in order to minimise the risk of losing the location. A possible explanation for the difference in scatter between the single point and array data could be the length of time the glass or silica surface was kept wet. The surface of the glass was not dried (to prevent the possibility of accidentally disturbing the lens) between each of the thirty measurements taken at single location. Therefore it is possible that the prolonged contact between the water and the glass caused ions to leach out of the glassy network, changing the properties of the glass surface in that area. As the measurements in an array necessitated the movement of the lens, there was only brief contact between the coupling fluid and any one location on the surface of the glass. However, although we note the possibility of a systematic difference between the two types of measurement because of exposure to a (neutral) aqueous environment, applying the T-test to the two air surface and tin surface datasets returns T-statistics of $T_{58}=$ 0.396 with $P=0.694$ and $T_{58}=0.407$ with $P=0.686$ respectively. Thus there is no significant difference between the repeated measurements made at a single point and those made in an array. We conclude therefore that there is no significant influence of exposure to water on the Rayleigh wave velocity.

\subsection{The Elastic Constants of Glass Surfaces}

We propose a simple model for the elastic properties of the glass surfaces in which there is a distinct surface layer of constant thickness and potentially different physical properties from the bulk. Such an approach has been used in the past by Pecorari et al who modelled the effect of surface damage on Rayleigh wave velocity in 
brittle materials [18] and by $\mathrm{Wu}$ et al who modelled the effects of residual stress [19]. For simplicity, we also assume that this surface layer is of sufficient thickness that any influence of the bulk material can be ignored and that the elastic properties of this surface layer are isotropic. Thus it is fully described by two independent elastic constants. Given these two assumptions, we can combine the results of the nanoindentation and $z$-contrast acoustic microscopy measurements to obtain both these constants (here we take Poisson's ratio and Young's modulus) for the air and tin sides of the float glass surfaces.

Extracting the elastic property values of materials by combining experimental data obtained from nanoindentation and acoustic microscopy has been reported in detail in an earlier publication [14]. This is achieved by combining equations $1-5$ and solving them simultaneously to obtain Poisson's ratio and Young's modulus. Figure 2 presents the data for the elastic constants of the glass surfaces obtained using nanoindentation and $z$-contrast acoustic microscopy. In both cases the single experimental measurement provides a reduced elastic modulus that is a combination of the two independent elastic moduli and can thus be represented as a locus in a parameter space defined by the two constants (here taken to be Poisson's ratio and Young's modulus). The intersection of the two loci defines the value for the elastic constants and provides a graphical method of solution. Although this result can be obtained numerically, the graphical method gives a better illustration of the experimental uncertainty. The greater statistical scatter of the Rayleigh wave velocity data is clear from the loci representing the standard error of the mean for both sets of measurements. The elastic constants of the air and tin sides of the float glass, determined by this combined method, are presented in Table 3 and show a difference between the two surfaces, with the tin surface showing a significantly reduced value of both Young's modulus and Poisson's ratio. 


\section{Discussion}

In this study we have used both nanoindentation and Rayleigh wave velocity measurements to determine the elastic properties of glass surfaces. Nanoindentation shows a stable value of the plane strain Young's modulus at depths $>500 \mathrm{~nm}$, that is somewhat greater than the bulk values supplied by the manufacturer and returns a lower value for the tin side of the sheet when compared with the air side. There is also a significant difference between the Rayleigh wave velocities of the two glass surfaces. On combining these two sets of measurements, we are able to determine the independent elastic constants of the surface. This combined measurement show a value for Young's modulus that is larger than that of the bulk properties of the glass and show a slightly smaller than expected value for Poisson's ratio. They also indicate a small but significant difference in elastic properties between the air and tin sides of the glass sheet. In reaching these conclusions we have made a number of assumptions that are discussed in more detail here.

Elastic modulus and hardness measurements of the glass, made using nanoindentation, show a dramatic indentation size effect; with the apparent elastic modulus increasing rapidly with decreasing indentation depth, for depths $<200 \mathrm{~nm}$. Recent studies by both Kese et al $[20,21]$ and Howell et al $[9,22]$ of the pile-up around nanoindentations made on float glass surfaces have indicated that the "Oliver and Pharr" method, which we have used to analyse our data [11], may underestimate the contact area and thus overestimate the contact stiffness, particularly at small indentation depths $<150 \mathrm{~nm}$. Through careful atomic force microscope (AFM) imaging of the residual indentations and determining more accurate contact areas at low indentation depths, 
both sets of authors calculated corrected values for the near surface mechanical properties and found a dramatic reduction in the indentation size effect, seen with both hardness and elastic modulus.

Allowing for this pile up around the indenter may explain the change in glass mechanical properties with depth seen in figure 1 , however it may not be sufficient to account for the value of Young's modulus found at indentation depths $>600 \mathrm{~nm}$ being greater than that of bulk glass measurements. Kese et al argued that pile up accounts for all the discrepancy and in their published data the nanoindentation elastic modulus measurement is identical to the bulk glass value [21]. Howell et al show nanoindentation derived Young's modulus values that are slightly larger than their quoted bulk glass properties for float glass samples but stated that the values are similar enough to be equivalent [22]. Gong et al measured the elastic properties of float glass surfaces using nanoindentation and critically reviewed the application of the Oliver and Pharr analysis. [23] They observed that nanoindentation returned a slightly larger value for the Young's modulus (assuming a Poisson's ratio of 0.23 ) than the value obtained from bulk specimens and attributed this to errors in the tip shape analysis used both by the original Oliver and Pharr method and in their proposed modification. However, our measurement of the Rayleigh wave velocity returns a larger than expected value for Young's modulus of the near surface region. This measurement method does not deform the surface region and suggests that the indentation methods used here and in the literature may be measuring a real difference between the properties of the surface regions and the bulk glass.

By combining nanoindentation and Rayleigh wave measurements, we have made an implicit assumption that both techniques probe equivalent regions of the glass 
surface. The stress distribution beneath a loaded indenter will decay to the third power of distance and therefore it samples the material property to a few multiples of the depth of indentation. The Rayleigh wave velocity measurements also interrogate the near surface region of a material. The Rayleigh wave velocity of approximately $3300 \mathrm{~ms}^{-1}$ was measured at $1 \mathrm{GHz}$, which results in a wavelength of about $3 \mu \mathrm{m}$. Rayleigh waves decay exponentially into the depth of the material with a critical length scale similar to the wavelength [13]; with soda lime glass the amplitude of the Rayleigh wave at a depth of $1 \mu \mathrm{m}$ is approximately $10 \%$ of the surface displacement. Thus the depth of material probed by Rayleigh wave velocity in this material is slightly smaller than is probed by indentation at penetration depths $>500 \mathrm{~nm}$,

As outlined earlier, there are a number of possible mechanisms that could explain the differences between the two glass surfaces. These are: diffusion of $\mathrm{Sn}^{2+}$ or $\mathrm{Sn}^{4+}$ ions into the side in contact with tin the float bath, a possible difference in fictive temperature, $T_{f}$, and finally a difference in the defect density on each surface. We consider the influence of Sn diffusion into the glass first. The chemical composition of the near surface region of commercial soda lime glass produced by the float process is not uniform and there are substantial variations in chemical composition on both the air and tin sides. An example chemical profile of the glass surface as determined by the manufacturer is provided in the supplementary material. The near surface regions of the glass are normally deficient in sodium which is lost during the annealing and fire glazing processes. The tin surface has very high $\mathrm{Sn}$ ion concentration close to the surface and this decays to about $2 \mathrm{wt} . \%$ at $200 \mathrm{~nm}$. There is also significant $\mathrm{Sn}$ contamination of the air side of the sheet at depths $<20 \mathrm{~nm}$. Thus the assumptions we have made concerning constant composition and homogeneous elastic properties are over-simplifications. It is possible to estimate the properties of the glass surface by 
reviewing the properties of bulk glass of comparable composition. The bulk properties of Sn-doped soda-lime glasses have been studied by Krohn et al and Ziemath et al $[3,4]$. Krohn reported an increase in elastic modulus with increasing Sn concentration [3] and showed that this was consistent with the predictions of a model developed by Makishima and Mackenzie[24]. Ziemath found an increase in hardness with increasing Sn concentration and also an increase in density. The influence of Sn-doping on mechanical behaviour was also reviewed by Howells et al who also came to the conclusion that increased Sn concentration tends to increase both elastic modulus and hardness [10]. This is the opposite trend to our results, which find that the glass surface in contact with the tin bath during manufacture has a significantly lower elastic modulus when compared with the air surface.

The Rayleigh wave velocity is controlled by both elastic properties and material density(equations 3 and 4). Thus local changes in material density must also be accounted for in discussing the difference in Rayleigh wave velocity between the two glass surfaces. The loss of $\mathrm{Na}$ is likely to result in a reduction in density, whereas the increase in Sn concentration is expected to lead to an increase in density [4]. In figure 3 we explore how a uniform change in density will influence the elastic properties of the tin side of the glass calculated from the Rayleigh wave velocity. As we can see, an increase in density implies an equivalent increase in Young's modulus at constant $v_{R}$. On comparing figure 3 with figure $2 \mathrm{a}$ we can see that at 2 wt. $\%$ Sn the Rayleigh wave velocities of the air and tin sides display very similar elastic properties. We should be cautious in giving great weight to this observation because the tin side density profile will show continuous variation with depth and figure 3 assumes a constant density. However, it is well known that the Rayleigh wave velocity is most strongly influenced by the very near surface regions of the material, hence the influence of local changes in 
material density near the surface cannot be ignored [25]. However, we note from figure 3 that if we include the nanoindentation measurements in our analysis, although the Young's modulus value of the tin side approaches that of the air side, the Poisson's ratio values diverge. We also note that these data do not fully correlate with the findings of Krohn who measured an increase in Young's modulus with increasing Sn content [3], which would predict an increase in Rayleigh wave velocity rather than the decrease we have measured. Thus the influence of a surface excess of $\mathrm{Sn}$ is expected to show two opposing influences on Rayleigh wave velocity from changes in local density and elastic stiffness. Measurements on bulk Sn doped glasses suggest that these effects although of opposite sign, will be of similar magnitude $[3,4]$.

It is well documented that the tin side of float glass is significantly weaker than the air side. Several studies have identified the critical defect size on the tin side to be about $20 \mu \mathrm{m}$ in depth with those on the air side being closer to $10 \mu \mathrm{m}$ [26-28].

However, it is not the size of the critical defect that influences the elastic properties of a surface but the total integrated effect of the population of defects of all sizes. To test the hypothesis that the reduction in elastic modulus found on the tin side of the float glass is caused by a larger density of defects, we explore the interaction between defect density and elastic properties. The elastic properties of cracked bodies have been the subject of considerable interest in both the materials science and geological materials community. Here we use an analysis by Zhao, Tandon and Weng [29], who derived a model based on the Eshelby equivalent inclusion [30], which predicts the elastic properties of materials containing defects that vary in shape from an idealised plane crack to spheres. In this model the bulk modulus, $K$, and shear modulus, $\mu$, of a solid containing a distribution of randomly oriented cracks are given by: 


$$
\begin{gathered}
\frac{K}{K_{o}}=\frac{1}{1+\frac{16\left(1-v_{o}^{2}\right)}{9\left(1-2 v_{o}\right)} \eta} \\
\frac{\mu}{\mu_{o}}=\frac{1}{1+\frac{32\left(1-v_{o}\right)\left(5-v_{o}\right)}{45\left(2-v_{o}\right)} \eta}
\end{gathered}
$$

Where the subscript $o$ identifies the appropriate elastic constant of the uncracked body; $\eta$ is the crack density parameter as introduced by Budiansky and O'Connell [31], which for circular cracks of negligible opening is given by:

$$
\eta=\frac{N r^{3}}{V}
$$

Here $N$ is the number of circular cracks of radius $r$ in a given volume, $V$.

Equation 6 is plotted in figure 4a along with Young's modulus and Poisson's ratio normalised by their uncracked values. Figure $4 \mathrm{~b}$ shows the effect of crack density on the Young's modulus and Poisson's ratio of float glass, if we take the elastic moduli of the air side as representing the crack-free state. We can see that a crack density parameter in the range $10^{-2}-2 \times 10^{-2}$ can account for a reduction in Young's modulus from 80.5 to 77.3 GPa and of Poisson's ratio from 0.20 to 0.19 . These defect density values are in agreement with those obtained by Pecorari et al, [18] who considered the scattering of Rayleigh waves by surface breaking defects. Pecorari predicted defect densities in the range of 2-4 per wavelength of the Rayleigh wave for similar reductions in Rayleigh wave velocity as found between our tin-side and air-side specimens. At $1 \mathrm{GHz}$ the Rayleigh wavelength, $\lambda_{R}$, on a glass surface will be about $3 \mu \mathrm{m}$ hence, assuming the defects sit in a square array with spacing $1 / 4 \lambda_{R}-1 / 2 \lambda_{R}$, Pecorari's results predict a surface defect density of approximately $4 \times 10^{11}-1.5 \times 10^{12} \mathrm{~m}^{-2}$ on the tin side of the glass 
which for $10^{-6} \mathrm{~m}$ deep circular cracks in a $3 \times 10^{-6} \mathrm{~m}$ surface layer results in a crack density parameter in the range $0.07-0.4$, which is consistent with our predictions made using the model of Zhao et al. [29]

Warren et al measured defect densities on float glass surfaces using a Hertzian indentation technique [8]. They reported a distribution of defect densities for different sizes of defect on the surface in the range of $10^{6}-10^{7} \mathrm{~m}^{-2}$, as shown in Figure 5. Using equation 7 with this data results in a crack density parameter in the range $5 \times 10^{-5}-$ $5 \times 10^{-6}$. It is clear from Warren's results that there is a higher density of defects on the tin surface than is found on the air surface of the glass sheet. This difference would appear to be consistent with our data for the elastic moduli of glass surfaces. However, it is possible to convert Warren's data into a crack density parameter by using equation 7 summed over all crack sizes, if we assume a fixed thickness for the damaged region. These measured crack densities are given in Table 4 (assuming that the damage zone has a depth in the range $10^{-6} \mathrm{~m}-10^{-5} \mathrm{~m}$ ) and are 3 orders of magnitude lower than the crack density parameter predicted by our analysis of the reduction in elastic properties and that of Pecorari. On comparing the crack density computed from Warren's results with the predicted change in elastic properties (figure 4), it is clear that these values are too low to significantly influence the elastic properties of the surface.

However, since the publication of Warren's paper, further analysis of the principles underlying the Hertzian indenation method have been reported. Using simulated crack distributions Roberts demonstrated that the Hertzian test does not select all crack sizes equally [32]. A combination of the elastic properties of the indenter, those of the surface under test, and the radius of the indenting sphere lead to an optimum crack depth for which the technique is most sensitive. Thus it is likely that the 
results of the Hertzian tests carried out by Warren et al, which showed a maximum defect population at a size of $2 \mu \mathrm{m}$, will not be sensitive to cracks of either significantly larger or smaller sizes. It is unlikely that there will be a significant number of cracks with defects larger than reported in figure 5, otherwise the mechanical properties of bulk glass would be seriously compromised. However, given the minor impacts and abrasions that will occur as a glass sheet passes over rollers, it is possible that there will be a significant population of smaller defects that are not counted by the Hertzian method, although altering the local elastic properties and thus detected by the acoustic and indentation methods. Nonetheless, it seems unlikely that there are sufficient defects present on the tin side of the glass sheet to solely account for the changes in elastic properties we have observed.

The final mechanism that might influence the mechanical properties of the glass surfaces is the influence of fictive temperature. Levelut et al [33] used Brillouin spectroscopy to probe the sound velocity in a range of float glass specimens where the value of $T_{f}$ was varied by extensive annealing cycles; Figure 7 shows a plot of their data. There is a significant influence of fictive temperature on sound velocity which supports earlier work on the effect of fictive temperature on sound velocity in other silica based glasses $[5,6]$. However, if we assume that Rayleigh wave velocity, $v_{R}$, will vary with $T_{f}$ in the same manner as longitudinal wave velocity, then the $3 \%$ difference in $v_{R}$ seen between the air-side and the tin-side of the glass (Table 3) would be equivalent to a difference in $T_{f}$ of about $100^{\circ} \mathrm{C}$ between the two sides of the glass sheet. The fictive temperature of soda lime float glass can be determined through infra red reflectivity measurements [34]; however the reported differences in reflectivity between the two sides of a float glass sheet are small and are not consistent with such a difference in fictive temperature. 
To summarise, there appears to be no individual mechanism that can consistently account for the differences in the mechanical properties that we have measured for the tin and air surfaces of the glass sheet. Comparing the differences we have found between the two surfaces with the effect of increasing Sn content on the bulk mechanical properties of glass finds no correlation. Although the computed defect density using continuum mechanics is consistent with that obtained using a scattering approach [18], the only independent measure of defect density found too low a concentration of large $(>2 \mu \mathrm{m})$ defects to account for the difference in mechanical properties [7]. The difference in fictive temperature required to account for the mechanical property difference is $100^{\circ} \mathrm{C}$ and this appears to be greater than possible differences reported through infra red spectroscopy.

\section{Conclusions}

The mechanical properties of the two surfaces of a float-glass sheet have significantly different elastic constants, with the air-side showing slightly larger values of both Young's modulus and Poisson's ratio. The elastic properties of the near surface regions of float glass, on both the tin- and air-sides are greater than the generally accepted values for the bulk properties of the glass. These results are obtained from two independent methods of measurement and we conclude that the both the difference between the surface and bulk properties and the difference between the properties of the air- and tin-sides of the glass are not artefacts of the measuring methods. None of the mechanisms proposed to account for the differences in mechanical properties between the tin- and air-sides can individually account for our observations. Measurement of bulk physical properties in tin doped glasses show that both Young's modulus and 
density increase with increasing tin content $[3,4]$. These represent opposite trends to the lower elastic modulus and lower Rayleigh wave velocity we have measured on the tin side of a float glass sheet. The fictive temperature of a glass is known to strongly influence its mechanical properties but the difference required between the two glass surfaces appears to be too great to be consistent with other measurements in the literature. Although an increase in surface defect density produces the required change in both surface elastic properties and Rayleigh wave velocity, the difference in defect density required to account for the difference in mechanical properties between the two sides of a glass sheet appears to be too great to be consistent with literature data of crack densities.

\section{Acknowlegements.}

We thank Paul Warren and Jonathan Williams of Pilkington Technology Centre for supply of materials and helpful discussion on our results and on the fictive temperature of glass. We would also like to acknowledge Steve Roberts of the University of Oxford for comments on the Hertzian indentation method. OG thanks the Engineering and Physical Sciences Research Council (EPSRC) and Pilkington Technology Centre for a CASE Studentship.

\section{References}

[1] Colombin L, Jelli A, Riga J, Pireaux JJ, Verbist J. J. Non-Cryst. Sol. $1977 ; 24: 253$ 
[2] Lamouroux F, Can N, Townsend PD, Farmery BW, Hole DE. J. Non-Cryst. Sol. 21997;12:232.

[3] Krohn MH, Hellmann JR, Mahieu B, Pantano CG. J. Non-Cryst. Sol. $2005 ; 351: 455$.

[4] Ziemath EC, Saggioro BZ, Fossa JS, J. Non-Cryst. Sol. 2005:351:3870.

[5] Vacher R, Delsanti M, Pelous J, Cecchi L, Winter A, Zarzycki J. J. Mater. Sci. 1974;9:829.

[6] Le Parc R, Levelut C, Pelous J, Martinez V, Champagnon B. J. Phys. Cond. Matter. 2006;18:7507.

[7] Warren PD, Pecorari C, Kolosov OV, Roberts SG, Briggs GAD. Nanotechnology 1996;7:295.

[8] Warren PD, Kolosov OV, Roberts SG, Briggs GAD. Nanotechnology 1996;7:288.

[9] Howell JA, Hellmann JR, Muhlstein CL. J. Non-Cryst. Sol. 2007;354:189.

[10] Kolluru PV, Green DJ, Pantano CG, Muhlstein CL. J. Amer. Ceram. Soc. 2010;93:838.

[11] Oliver WC, Pharr GM. J. Mater. Res. 1992;7:64.

[12] Kushibiki J, Chubachi N. IEEE Trans. Sonics and Ultrasonics 1985;SU32:189.

[13] Landau LD, Lifshitz EM. Theory of Elasticity: 3rd Edition, Butterworth Heinemann, Oxford, UK, 1986.

[14] Bamber MJ, Cooke KE, Mann AB, Derby B. Thin Solid Films 2001;398:299.

[15] Pilkington. Technical Information - properties of soda-lime-silica float glass. St. Helens, UK, 1999, 1. 
[16] ISO 14577-4:2007 Metallic Materials- Instrumented Indentation Tests forHardness and Materials Parameters, Part 4: Test Methods for Metallic and Non-Metallic Coatings. ISO, Geneva, Switzerland, 2007.

[17] Cordill MJ, Moody NR, Gerberich WW. J. Mater. Res. 2008;23:1604.

[18] Pecorari C, Lawrence CW, Roberts SG, Briggs GAD. Phil. Mag. A 2000;80:2695.

[19] Wu HZ, Roberts SG, Derby B, Acta Mater. 2008;56:140.

[20] Kese KO, Li ZC, Bergman B. J. Mater. Res. 2004;19:3119.

[21] Kese KO, Li ZC, Bergman B. Mater. Sci. Eng. A 2005;404:1.

[22] Howell JA, Hellmann JR, Muhlstein CL. Mater. Lett. 2008;62:2140.

[23] Gong J, Hezhuo M, Zhijian P. Acta Mater. 2004;52:785.

[24] Makishima A, Mackenzie JD, J. Non-Crys. Solids 1973;12:35.

[25] Tiersten HF. J. Appl. Phys. 1969;40:770.

[26] Entwistle KM. J. Mater. Sci. 1993;28:2007.

[27] Krohn MH, Hellmann JR, Shelleman DL, Pantano CG, Sakoske GE. J. Am. Ceram. Soc. 2002;85:1777.

[28] S.T. Gulati, R. Akcakaya, J.R. Varner, Ceram. Trans. 122 (2001) 317.

[29] Zhao YH, Tandon GP, Weng GJ. Acta Mech. 1989;76:105.

[30] Eshelby JD. Proc. Roy. Soc. Lon. 1957;241:376.

[31] Budiansky B, O'Connell RJ, Inter. J. Solids Structures 1976;12:81.

[32] Roberts SG. Brit. Ceram. Trans. 2000;99:31.

[33] Levelut C, Le Parc R, Faivre A, Champagnon B., J. Non-Cryst. Sol. 2006;352:4495.

[34] Ararwal A, Tomozawa M. J. Amer. Ceram. Soc. 1978;78:827. 


\section{Figure Captions}

Figure 1. Mechanical property data determined as a function of indentation depth into the surface of the air side (square symbols) and tin side (round shaded symbols) of a float glass specimen. a) Plane strain Young's modulus $\left(E^{*}=1 /\left(1-v_{R}^{2}\right)\right)$; b) Hardness (Berkovich indenter). In both cases the error bars indicate one standard deviation of the data.

Figure 2 Data from nanoindentation and $V(z)$ curves can be represented as curves in a parameter space defined by Young's modulus, $E$ and Poisson's ratio, $v$. Intersection of the curves defines the two independent elastic constants of the glass surface: a) air-side, b) tin side. Dashed and dotted lines represent the standard error of the experimental data used to generate the curves.

Figure 3 A comparison of how surface concentrations of Sn ions influence the bulk density and hence the calculated

Figure 3 The influence of a randomly oriented crack distribution on the elastic constants of solids using the model of Zhao et al [17]. a) Change in elastic constants, normalised by the uncracked values, as a function of crack density. b) Predicted change in the Young's modulus, $E$, and Poisson's ratio, $v$, of float glass as a function of crack density.

Figure 4 The defect density on soda-lime float glass surfaces as measured using Hertzian indentation. Data plotted from values reported by Warren et al. [8]. 
Figure 5 The room temperature longitudinal sound velocity as a function of fictive temperature for a soda-lime float glass. Data plotted from values reported by Levelut et al [22]. 
Table 1. Nominal composition and properties of the float glass specimens as supplied by Pilkington Technology Centre [15].

\begin{tabular}{|l|l|l|l|l|}
\hline Component & Wt\% & Mole\% & Property & \\
\hline $\mathrm{SiO}_{2}$ & 72.6 & 71.7 & Density & $2530 \mathrm{kgm}^{-3}$ \\
\hline $\mathrm{Al}_{\mathbf{2}} \mathbf{O}_{3}$ & 1.0 & 0.6 & Young's Modulus & $73 \mathrm{GPa}$ \\
\hline $\mathrm{Na}_{\mathbf{2}} \mathbf{O}$ & 13.0 & 12.5 & Poisson's Ratio & 0.23 \\
\hline $\mathrm{K}_{\mathbf{2}} \mathbf{O}$ & 0.6 & 0.4 & & \\
\hline $\mathrm{MgO}$ & 3.9 & 5.7 & & \\
\hline $\mathbf{C a O}$ & 8.4 & 8.9 & & \\
\hline $\mathbf{F e}_{2} \mathbf{O}_{3}$ & 0.10 & 0.04 & & \\
\hline $\mathbf{S O}_{3}$ & 0.2 & 0.2 & & \\
\hline
\end{tabular}


Table 2. Mean Rayleigh wave velocities and their standard error, measured from a silica reference specimen and the air and tin surfaces of $4 \mathrm{~mm}$ thick float glass, calculated from 30 different $z$-contrast measurements taken either at a single point or from a 5 x 6 array of points..

\begin{tabular}{|l|l|l|}
\hline Sample & Number of & $\begin{array}{l}\text { Mean } \boldsymbol{v}_{\boldsymbol{R}} \\
\left(\mathrm{ms}^{-1}\right)\end{array}$ \\
\hline & Seasurements & \multicolumn{2}{|l|}{} \\
\hline Silica & 30 & $3386 \pm 22$ \\
\hline Air & 30 & $3323 \pm 22$ \\
\hline Tin & 30 & $3386 \pm 28$ \\
\hline & Array Measure \\
\hline Silica & 30 & $3312 \pm 17$ \\
\hline Air & 30 & $3252 \pm 20$ \\
\hline Tin & 30 & \\
\hline
\end{tabular}


Table 3 The elastic constants of the air and tin surfaces of a sheet of float glass as determined by a combination of nanoindentation and $z$-contrast acoustic microscopy.

\begin{tabular}{|l|l|l|}
\hline Surface & $\begin{array}{l}\text { Young's } \\
\text { Modulus }\end{array}$ & Poisson's \\
& Ratio \\
\cline { 2 - 3 } & $(\mathrm{GPa})$ & \\
\hline Glass - Air side & $80.5 \pm 0.5$ & $0.20 \pm 0.01$ \\
\hline Glass - Tin side & $77.3 \pm 1.0$ & $0.19 \pm 0.015$ \\
\hline
\end{tabular}

Table 4 Crack density parameter calculated from the data of Warren et al [8] assuming cracks are concentrated in a region of defined depth.

\begin{tabular}{|l|l|l|l|l|}
\hline \multirow{2}{*}{ Depth of Region } & \multicolumn{4}{|c|}{ Crack Density Parameter } \\
\cline { 2 - 5 } & $10^{-6} \mathrm{~m}$ & $3 \times 10^{-6} \mathrm{~m}$ & $6 \times 10^{-6} \mathrm{~m}$ & $10^{-5} \mathrm{~m}$ \\
\hline Glass - Air side & $7.8 \times 10^{-6}$ & $1.3 \times 10^{-5}$ & $2.6 \times 10^{-5}$ & $7.8 \times 10^{-5}$ \\
\hline Glass - Tin side & $3.9 \times 10^{-6}$ & $6.5 \times 10^{-6}$ & $1.3 \times 10^{-5}$ & $3.9 \times 10^{-5}$ \\
\hline
\end{tabular}


a)

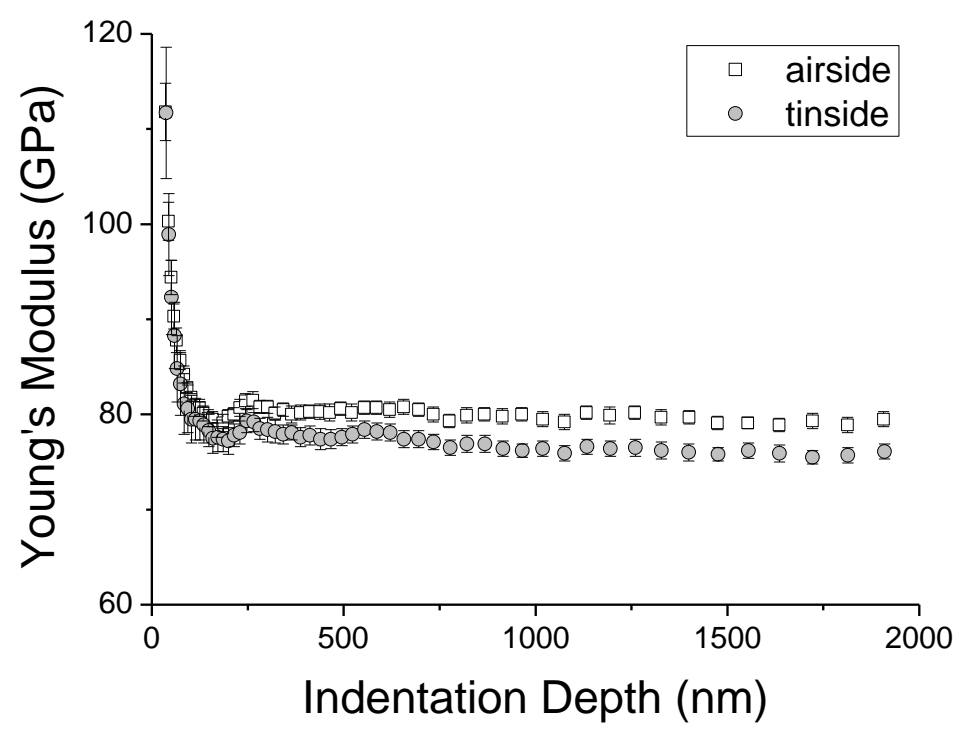

b)

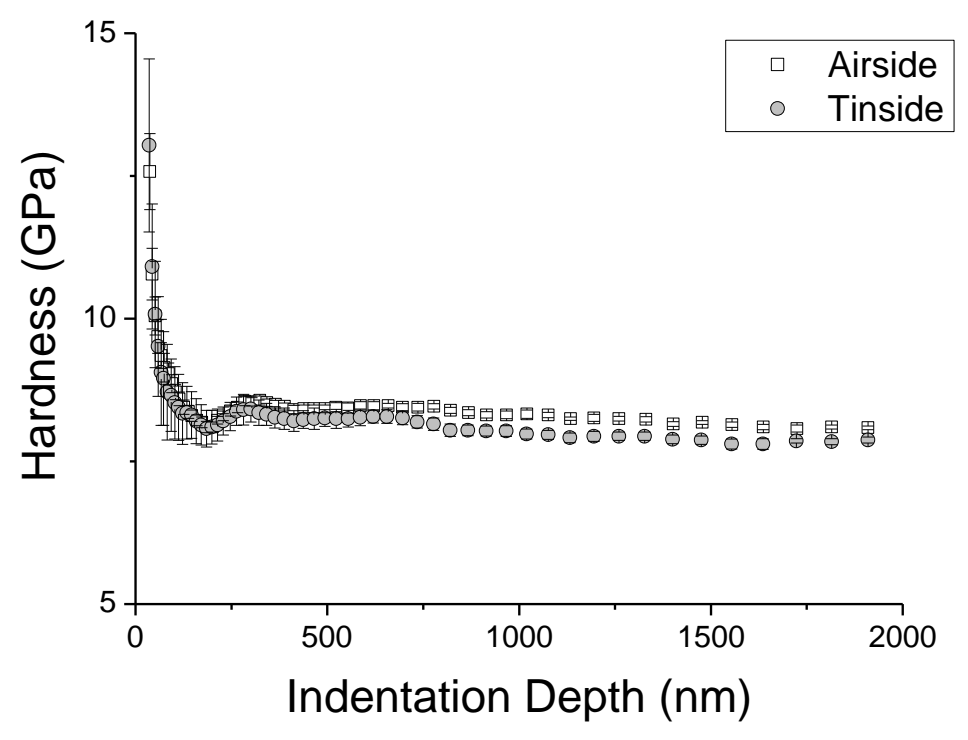

Figure 1 Nanoindentation data as a function of indentation depth into the surface of the air side (square symbols) and tin side (round shaded symbols) of a float glass specimen. a) Young's Modulus (with an assumed Poisson's ratio of 0.23); b) hardness (Berkovich indenter). In both cases the error bars indicate one standard deviation of the data. 
a)

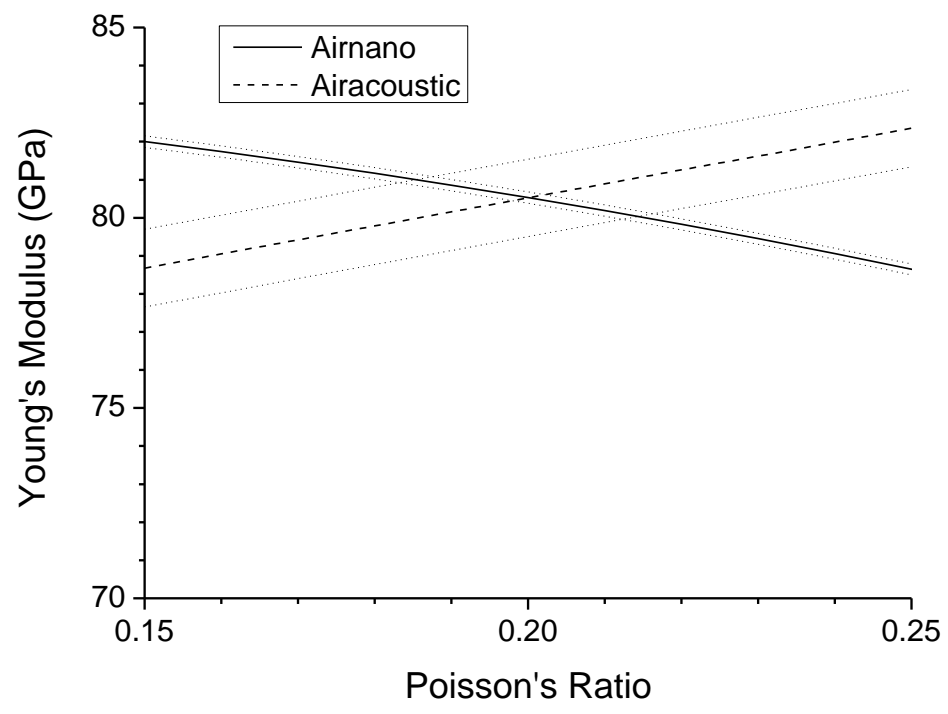

b)

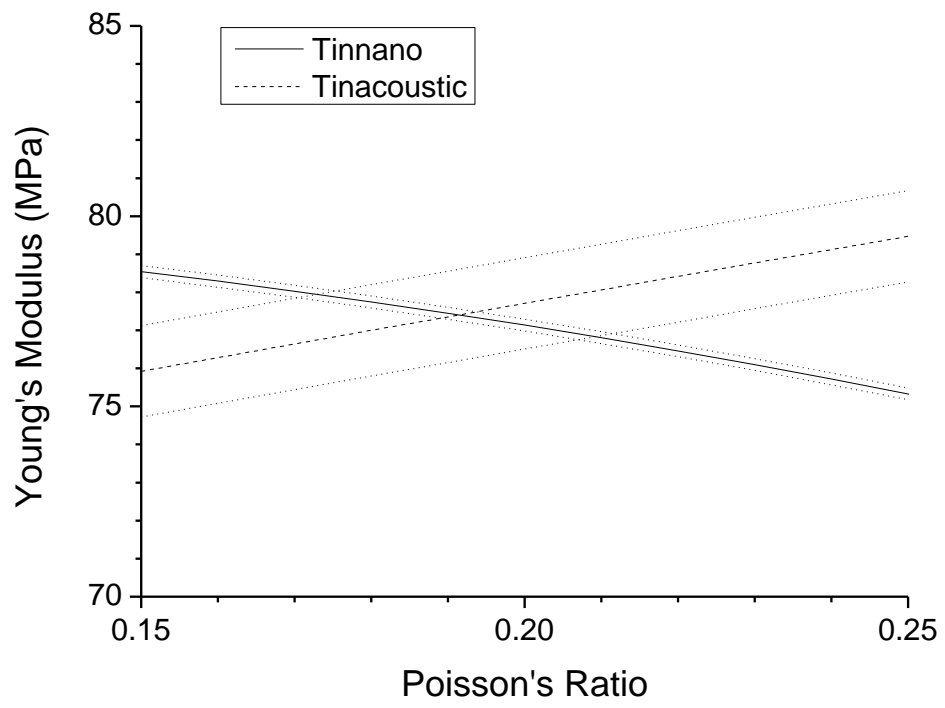

Figure 2 Data from nanoindentation (solid lines) and $V(z)$ curves (dashed lines) can be represented as curves in a parameter space defined by Young's modulus, $E$ and Poisson's ratio, $v$. Intersection of the curves defines the two independent elastic constants of the glass surface: a) air-side, b) tin-side. Dotted lines represent the standard error of the experimental data used to generate the curves. 


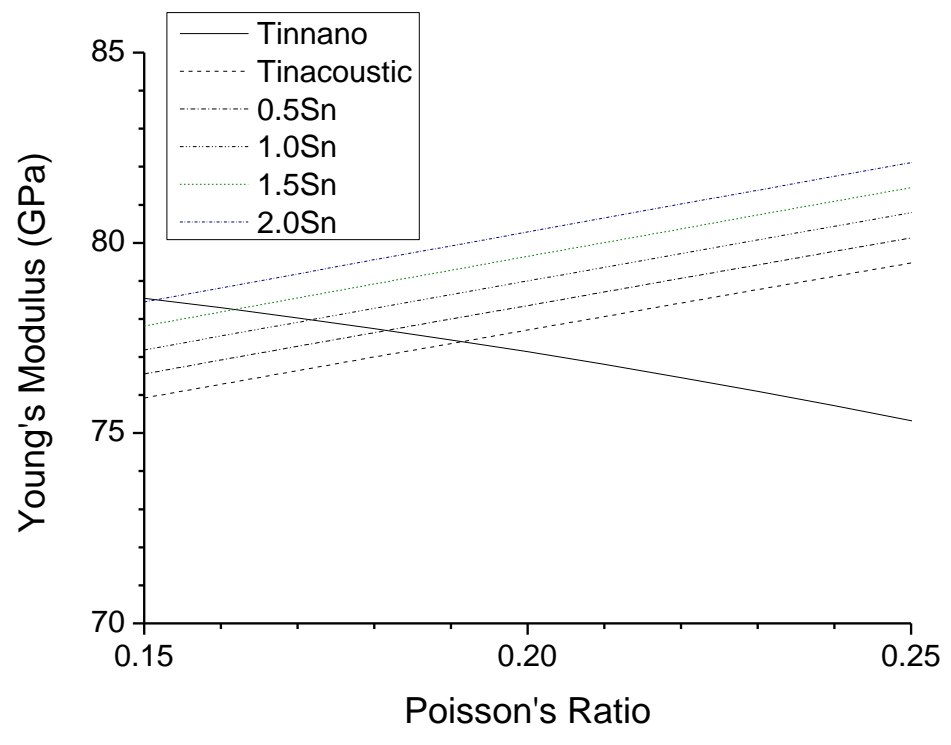

Figure 3 Influence of increase in density on elastic properties determined from Rayleigh wave velocity measurements. Solid line indicates the locus of elastic properties determined from the tin side of the glass sheet using nanoindentation. The dashed lines represent a series of elastic property measurements made from the Rayleigh wave velocity assuming different surface densities estimated from the values of bulk Sn-doped glass as reported by Ziemath [4]. 
a)

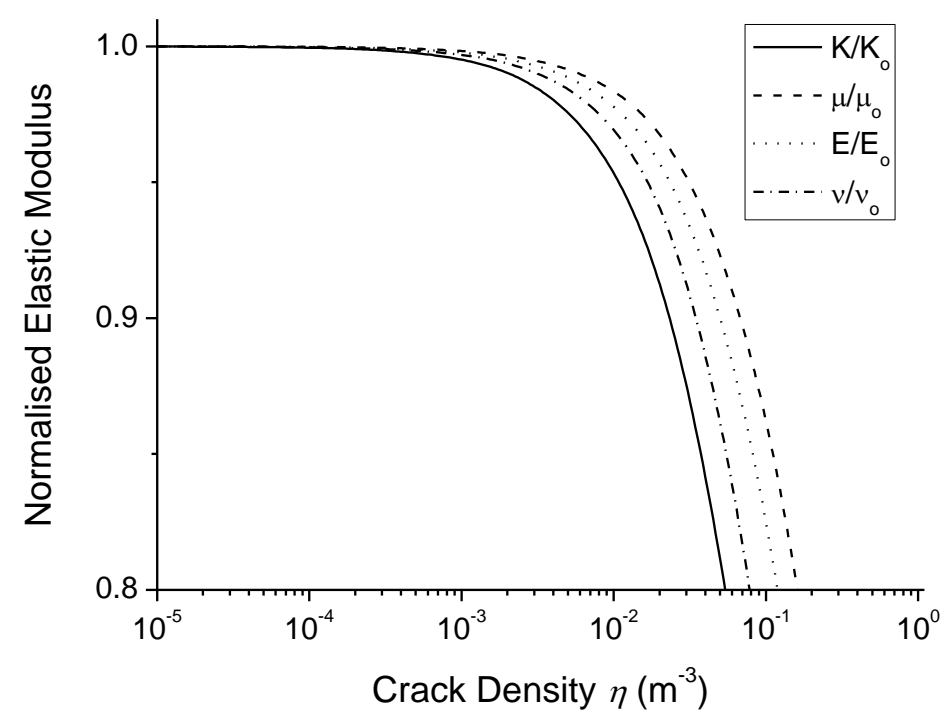

b)

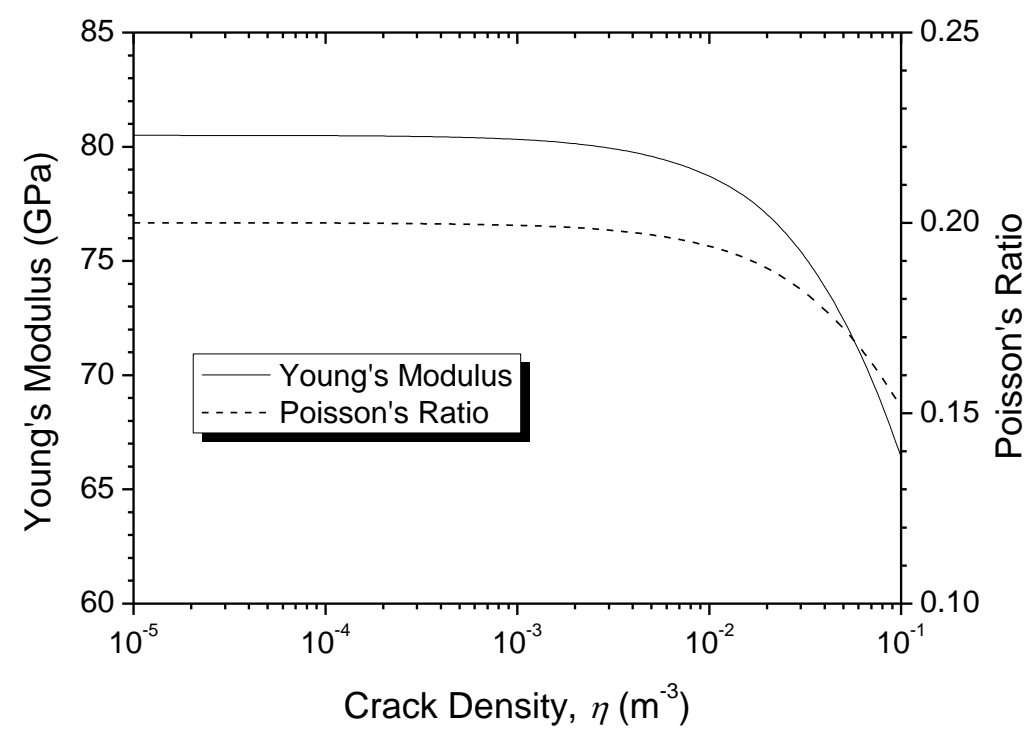

Figure 4 The influence of a randomly oriented crack distribution on the elastic constants of solids using the model of Zhao et al [17]. a) Change in elastic constants, normalised by the uncracked values, as a function of crack density. b) Predicted change in the Young's modulus, $E$, and Poisson's ratio, $v$, of float glass as a function of crack density. 


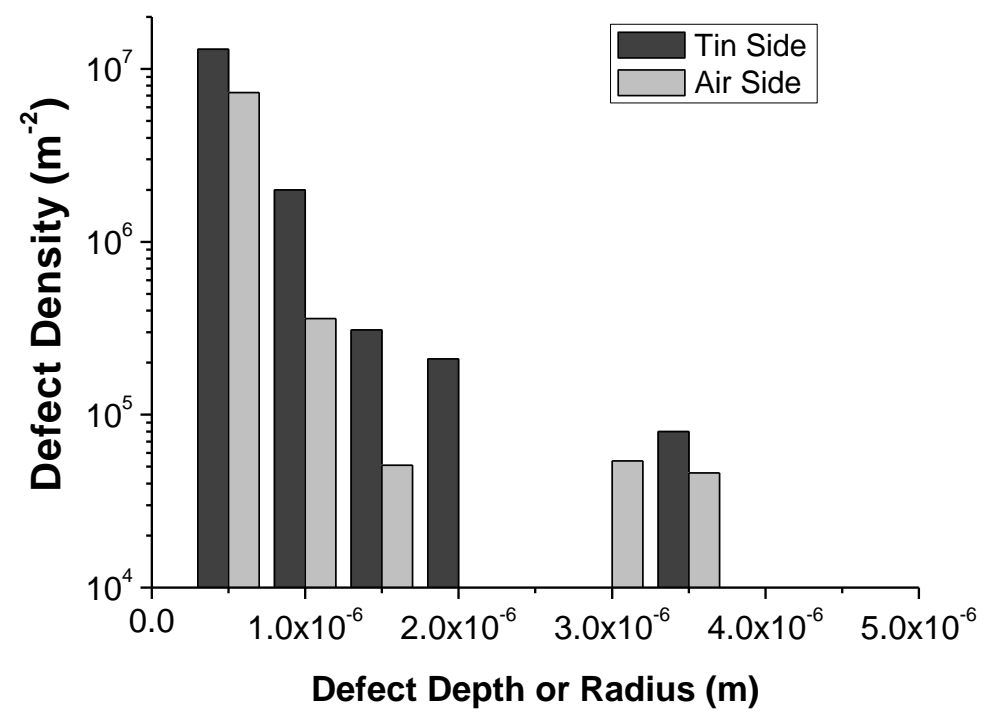

Figure 5 Histogram showing the defect density on soda-lime float glass surfaces as measured using Hertzian indentation. Data plotted from values reported by Warren et al. $[8]$. 


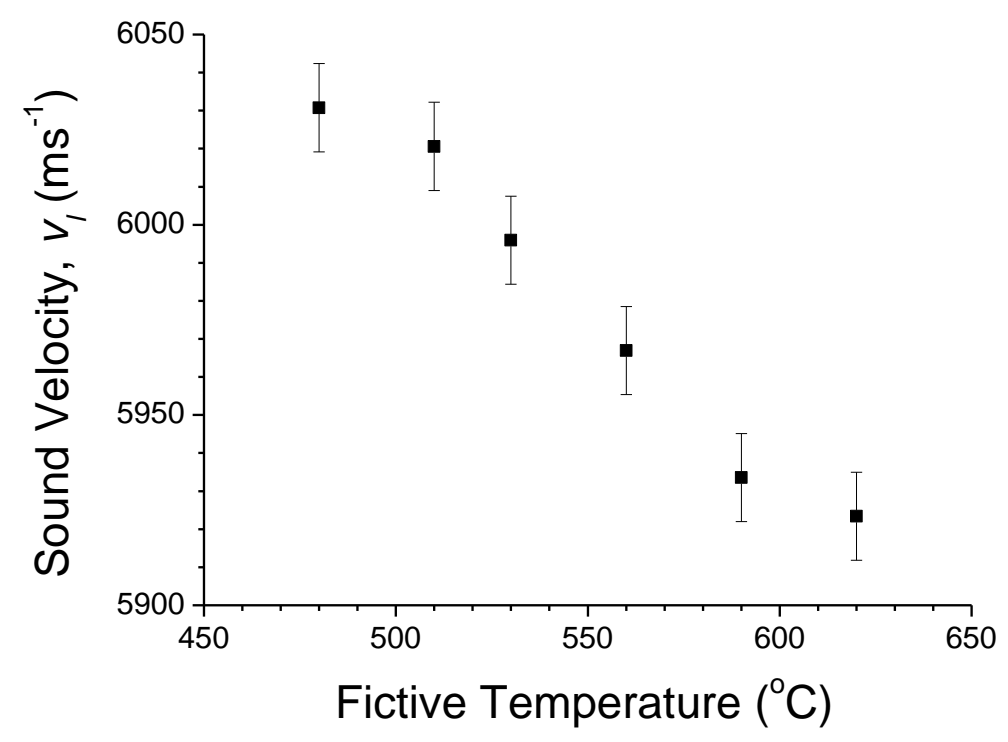

Figure 6 The room temperature longitudinal sound velocity as a function of fictive temperature for a soda-lime float glass. Data plotted from values reported by Levelut $e t$ al [22]. 